\title{
Functional ectodomain of the hemagglutinin-neuraminidase protein is expressed in transgenic tobacco cells as a candidate vaccine against Newcastle disease virus.
}

\begin{abstract}
Recently, the use of plants for the production of recombinant proteins has been well demonstrated with promising outcomes. In this study, an efficient Nicotiana tabacum L. cv. Bright Yellow 2 (BY-2) cells system expressing the ectodomain of hemagglutininneuraminidase (eHN) protein from Newcastle disease virus (NDV) strain AF2240 was established. Transgenic tobacco BY-2 cell cultures expressing the immunogenic eHN protein were generated and the translation efficiency of eHN protein was enhanced using the 5'untranslated region of Nicotiana tabacum alcohol dehydrogenase gene (NtADH 5'-UTR) under the control of strong cauliflower mosaic virus (CaMV 35S) promoter. Transgenic lines verified by real-time PCR showed high level of eHN mRNA transcripts and immunoblotting confirmed the presence of $66 \mathrm{kD}$ eHN protein. The eHN protein was stably produced in an average of $0.2-0.4 \%$ total soluble protein. Green fluorescent protein-tagged eHN protein was expressed and localized at the cytosol of BY-2 cell. All mice receiving purified eHN protein from transgenic tobacco BY-2 cells produced specific anti-NDV antibodies. We concluded that plant made eHN elicit immune response and can serve as candidate vaccine against NDV.
\end{abstract}

Keyword: Hemagglutinin-neuraminidase; Newcastle disease virus; NtADH 5'-UTR; Plantbased vaccine. 